\title{
The Link between Facets of Impulsivity and Aggression in Extremely Violent Prisoners
}

\author{
Henning Værøy ${ }^{1}$, Elin Western'2, Stein Andersson ${ }^{3}$ \\ ${ }^{1}$ Department of Psychiatric Research and Development, Akershus University Hospital, Loerenskog, Norway \\ ${ }^{2}$ Department of Pediatric Neuroscience, Oslo University Hospital, Rikshospitalet, Norway \\ ${ }^{3}$ Institute of Psychology, University of Oslo, Oslo, Norway \\ Email: *Henning.Vaeroy@ahus.no
}

Received 19 November 2015; accepted 16 January 2016; published 19 January 2016

Copyright (C) 2016 by authors and Scientific Research Publishing Inc.

This work is licensed under the Creative Commons Attribution International License (CC BY).

http://creativecommons.org/licenses/by/4.0/

(c) (i) Open Access

\section{Abstract}

Evidence is growing that aggressive behavior and impulsivity have subgroups. The subscales of the Urgency, Premeditation, Perseverance and Sensation seeking (UPPS) impulsivity scale and the Bryant and Smith shortened and refined version of the Aggression Questionnaire were used to describe and compare impulsive and aggressive behavior in extremely violent and aggressive male inmates and non-violent healthy male controls. The Mann-Whitney test showed that there was a significant difference $(p<0.006)$ in the total UPPS impulsivity scale scores between the aggressive inmates and the controls. The subscales revealed that this difference was based mainly on the urgency score $(p<0.003)$. On the aggression subscales, the inmates scored significantly higher for physical aggression than the controls $(p<0.001)$, but no significant difference was seen between inmates and controls for verbal aggression, anger and hostility, although the exact $p$ value was very close to statistical significance at 0.054 . Regression analysis revealed a strong relationship between urgency and the aggression subscales hostility $(p=0.0004)$ and anger $(p=0.003)$ and that urgency was also linked to symptoms of anxiety $(p=0.008)$. Finally, a statistically significant link was found between both hostility $(p=0.0003)$ and anger $(p=0.002)$ and symptoms of anxiety. The highly selected subgroup of extremely violent criminals in this study were more physically aggressive than non-violent controls, with urgency as the driving feature in their impulsive behavior, and hostility, anger and symptoms of anxiety as underlying traits.

\section{Keywords}

UPPS, Aggression, Impulsivity, Inmates, Prisoners

\footnotetext{
${ }^{*}$ Corresponding author.
}

How to cite this paper: Værøy, H., Western, E. and Andersson, S. (2016) The Link between Facets of Impulsivity and Aggression in Extremely Violent Prisoners. Open Journal of Psychiatry, 6, 86-94. 


\section{Introduction}

The biological complexity of the development of aggression is partly illustrated by studies showing that childhood trauma and genetic polymorphism may interact and increase the risk for aggressive behavior in male prison populations [1]. Lower amygdala volume is associated with male aggression and the development of future violence in children [2]. Changes in testosterone levels are known to modulate aggressive behavior, and decreasing levels of cortisol over time are a predictor for persistent aggression in highly aggressive adolescents [3]. Psychologically, competition may in some cases represent a situation in which aggressive behavior may follow, but only in men with low trait anxiety [4]. Attempts to classify aggression have been made over the years, and impulsive (reactive) aggression as a disorder has been included in the DSM diagnostic system since the first edition [5] [6]. Impulsive aggression seems heritable by genetic transmission [7] and it is strongly predicted by cognitive and affective empathy [8]. Impulsive aggression does not predict violence recidivism as opposed to premeditated aggression [9]. Impulsive aggression often occurs in response to perceived provocation [10] whereas premeditated or proactive aggression is goal-directed, and carried out unemotionally [11] [12].

The relationship between impulsivity and aggression has long been established and is recognized in terms such as "impulsive aggression". Less is known about the relationship between impulsivity and anxiety. Some claim that both have clear relations to aggression [13]. Others see anxiety as a possible mediator in settings where both impulsivity and aggression interact [14] or conclude that impulsivity has the same effect on aggression, in this case physical, regardless of the level of anxiety [15]. Hostile impulses have been included among known risk-prone activities for social anxiety disorder in response to impulsive decision-making [16]. Impulsivity has been linked to 2 different classes of social anxiety disorder, one of which is associated with higher levels of anger, aggression, sexual impulsivity and substance abuse [17] [18].

It is not known why some socially anxious individuals are vulnerable to become aggressors, but culture and honor-concerns are known to influence this, as can a feeling of being persecuted, since the latter is significantly linked to an aggressive attitude [19]. Angry or anxious persons display a more aggressive attitude than persons in a normal mood state [20] and anxiety is most likely the mediator when, for example, physical and sexual aggression towards a partner are used, as in cases of anticipated infidelity [14].

Several theories have been proposed to explain and understand impulsive behavior, but much is still unclear [21]-[25].

In Emil Kraepelin's Lehrbuch der Psychiatrie (Textbook of Psychiatry, 1904) [26], the term impulsive insanity refers to often purposeless, unpremeditated, irresistible impulses, often morbid, to destroy and kill. In fact, there is a high prevalence of both recurrent suicide attempts (87\%) and hypomanic episodes (62\%), especially where impulsivity co-exists with anxiety [27].

Perhaps the best known and most frequently used scale for measuring impulsivity is the Barratt Impulsiveness Scale (BIS), [28]-[32]. The Five-Factor Model of Personality [33] introduces the possibility to better understand and position the different concepts of impulsivity, and to add clarity to the assortment of impulsivity measures. This led to the development of the UPPS impulsivity scale [34] which focuses on facets of impulsivity common across the different measures.

We thought that the Buss Perry Aggression Questionnaire (AQ) [35] in its Swedish version, the Aggression Questionnaire in the Revised Swedish Version (AQ-RSV), [36], and Bryant and Smith-refined Aggression Questionnaire subscales (BS-rAQ) [37] would reveal important nuances in our population of extremely aggressive individuals, thereby providing us with new information.

In the present study the impulsivity subscales urgency, premeditation, perseverance and sensation seeking were applied together with the aggression subscales hostility, physical and verbal aggression and anger. The aim of the study was to see how the different subscales performed against each other in a highly selected population of extremely violent inmates and to establish whether the use of subscales provided new information. We were unable to find any other publications on a similar population assessed using the subscales we applied here.

\section{Methods}

This study was conducted in two stages. Recruitment of the inmates and the first group of controls was done in 2011 and 2012 and the second control group in 2014. 


\subsection{Study Population}

\subsubsection{Inmates}

To be included as a study subject in this study a long-term sentence due to extremely violent crime was a prerequisite. Sixteen violent male inmates, mean age $41.9+11.9$ (SD) years, the majority in preventive detention, were recruited from a high security prison outside Oslo, except for one who had recently been released but still agreed to participate. He was later re-arrested and charged following new episodes with violent behaviour. Fifteen of the 16 violent inmates were assessed using the AQ-RSV [36] and the UPPS [34]; one inmate did not complete the questionnaire. Eleven of the16 inmates had committed at least one murder or had attempted to commit murder, and 4 had committed severely violent crimes with a sex-related component. The main features of the extremely brutal violence were rape, molestation or grievous bodily harm. Inmates diagnosed with paedophilia were not included in this study. One inmate had participated in gang-related violence, killing one person.

Fifteen of the inmates were serving long-term sentences, the majority in preventive detention.

In Norway, the imposition of preventive detention indicates that the court considers the defendant at a high risk for reoffending, and therefore as an imminent threat to society. According to Norwegian law, after having served a minimum term not exceeding 10 years, prisoners in preventive detention may ask the court to reconsider their case.

Before inclusion, the inmates were thoroughly informed about the inclusion and exclusion criteria. Among the 15 prisoners, no serious mental illness was reported or found when clinically screened for psychiatric history and current disorders at the time of the first visit.

The process of recruiting inmates who have committed very violent and aggressive acts is complex and challenging. Furthermore, in Norway, the total number of excessively violent inmates is much smaller than in countries with large populations. Severe security measures usually apply and the inmates who eventually agreed to participate represent a highly selected population. In the present study, the 15 high security level inmates were assessed using the AQ-RSV [36]. Having identified and extrapolated the 12 questions in the AQ-RSV that correspond to those forming the BS-rAQ [37], this paper presents the BS-rAQ scores from 15 extremely violent inmates sentenced to long-term imprisonment, and their scores on a translated, authorized, but unpublished, Norwegian version of the UPPS impulsivity scale [34].

\subsubsection{Controls}

In this study, two healthy male control (HC) groups were randomly recruited from the general population. The first HC group (HC-I) of 21 subjects, mean age $41.8+10.4$ (SD) years old, was recruited at an early stage of the study. The second group (HC-II) of 37 healthy males was recruited as controls for the UPPS. The mean age of the HC-II group was $39.5+10.1$ (SD) years. The HC-II group was also assessed using the BS-rAQ and the Hospital Anxiety and Depression Scales (HAD), and was added to the already existing healthy male control group, HC-I. Consequently, aggression and anxiety scores from an HC group of 58 subjects were available for comparison. None of the control subjects had any history of violence and reported no prior or concurrent psychiatric or neurological disorders.

\subsection{Questionnaires}

The self-rating Buss-Perry Aggression Questionnaire (AQ) [35] with its 4 subscales describing verbal and physical aggression, anger, and hostility is extensively used and has been translated into several languages, including a revised Swedish version (AQ-RSV) [36].

1) The Aggression Questionnaire-Revised Swedish Version (AQ-RSV)

The Swedish version of the AQ [36] was developed for research into clinical aggression based on the US version of the AQ [35]. For validation, the test was mailed to 781 randomly selected individuals aged 20 - 40 years. Of these, scores from 497 test persons were evaluable (64\%). The results from the American and the Swedish versions were comparable for the correlations between the four aggression subscales and had alpha coefficients indicating internal consistency. The Swedish and Norwegian cultures and languages are very close, which means that we did not expect any major problems applying the AQ-RSV directly to a Norwegian population.

2) The Bryant and Smith shortened and refined Aggression Questionnaire (BS-rAQ).

Bryant and Smith [37] introduced a 12-question refined measurement model (BS-rAQ) of the original AQ 
[35]. Due to overlap between what the subscales actually measured and low common variance, the AQ was consequently refined by Bryant and Smith [37].

The inmates' individual AQ-RSV scores corresponding to the 12 questions forming the BS-rAQ were selected for the BS-rAQ subscale analysis in this study.

3) The UPPS impulsivity scale.

Whiteside and Lynam [34] identified 4 distinct facets of personality that had been frequently confused and commonly referred to as impulsivity. These facets, urgency, premeditation, perseverance and sensation-seeking, form the UPPS impulsivity behavior scale [34].

The Hospital Anxiety and Depression (HAD) scale [38] is a 14 item self-report measure with two subscales, each of 7 items for detecting states of depression and anxiety. In this study, to qualify for clinically significant anxiety and depression, a cut-off point of $>8$ was chosen for both the anxiety and depression subscales.

The inmates were assessed using the UPPS [34], the AQ-RSV [36] and the HAD scale sitting at a table in a room in the visiting area outside their departments at the prison.

\subsection{Statistics}

All statistical analyses (linear and non-linear regression analysis, Mann-Whitney [MW] test, Student's t-test and the Kolmogorov-Smirnov [KS] test) were performed using GraphPad Software, Inc., La Jolla, CA 92037 USA. A $p$-value $<0.05$ was considered statistically significant.

\subsection{Ethics}

The study was approved by the Norwegian Regional Ethics Committee-approval number 2010/792.

\section{Results}

\subsection{Mann-Whitney and Kolmogorov-Smirnov Tests}

The MW test showed a significant difference $(p<0.006)$ in the total UPPS impulsivity scale scores between the aggressive inmates and the HC-II group. The result in the KS test was $p<0.003$. Looking at the 4 subscales, only urgency showed a statistically significant difference $(p<0.003)$ in the MW test, and a p-value of 0.02 in the KS test. There were no differences with a $p$-level $\leq 0.05$ between inmates and HC-II controls for the remaining 3 UPPS subscales.

The total number of controls who completed the BS-rAQ was 58. The MW test showed that the inmates were significantly more physically aggressive than the controls $(p<0.001)$ and the KS test gave a $p$-value of 0.002 for the same difference. No significant difference was seen for the subscales verbal aggression, anger and hostility in the MW test, but for hostility the exact $p$-value of 0.054 was very close. The KS test, in this case, gave a $p$-value of 0.140 . Furthermore, the KS test showed statistical significance (0.047) for anger but not for verbal aggression.

\subsection{Regression Analysis}

\subsubsection{Impulsivity and Aggression}

Linear regression analyses between the inmates’ urgency scores and hostility and anger yielded statistically significant relationships (Figure 1).

\subsubsection{Impulsivity and Symptoms of Anxiety}

Figure 2 displays the linear regression between the inmates' individual urgency scores and HAD A subscale scores for anxiety $(p=0.008)$. No statistically significant relationship was seen between any UPPS impulsivity scales and depression, or between the anxiety scores and the 3 remaining UPPS subscales.

\subsubsection{Aggression and Symptoms of Anxiety}

Linear regression between hostility and anger and the HAD A subscale also yielded statistically significant relationships as seen in Figure 3(a) (hostility $p=0.0003$ ) and Figure 3(b) (anger $p=0.002$ ).

Four inmates scored equal to or above the cut-off score of $\geq 8$ on the anxiety subscale and 1 inmate scored 


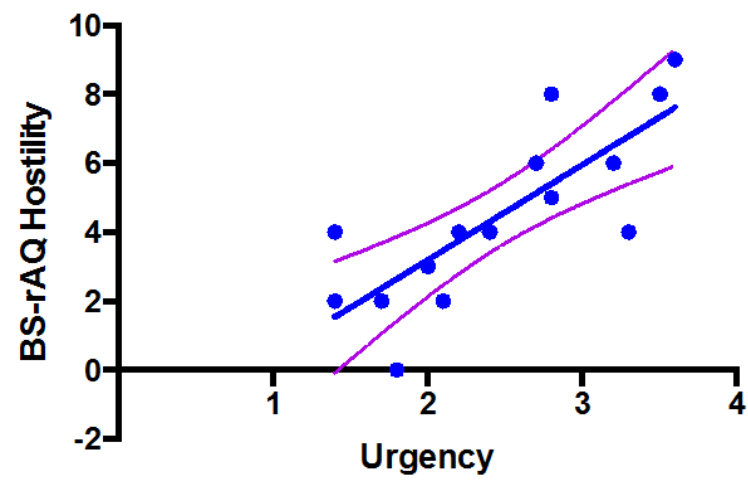

(a)

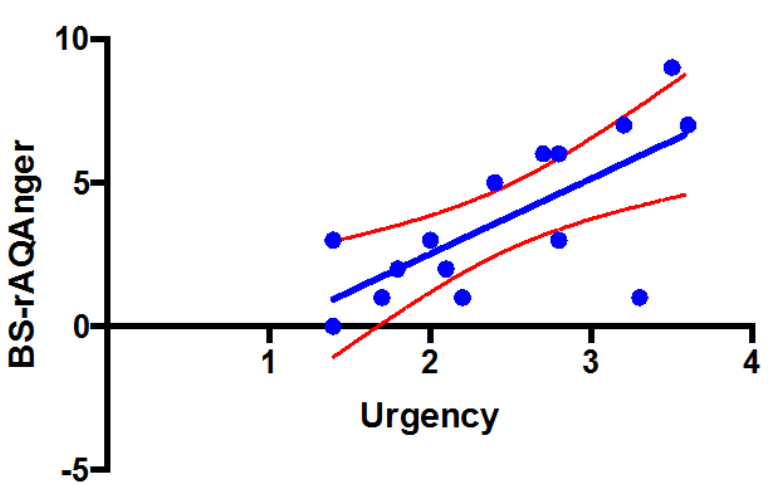

(b)

Figure 1. (a) Displays the linear regression between the inmates' urgency and hostility scores; $p=0.0004, \mathrm{Y}=2,762 * \mathrm{X}-$ 2,328. (b) Displays the linear regression between the inmates' urgency and anger scores; $p=0.003, \mathrm{Y}=2,625^{*} \mathrm{X}-2,724$. Linear regression revealed no statistically significant relationships between premeditation, perseverance, sensation-seeking, and physical and verbal aggression.

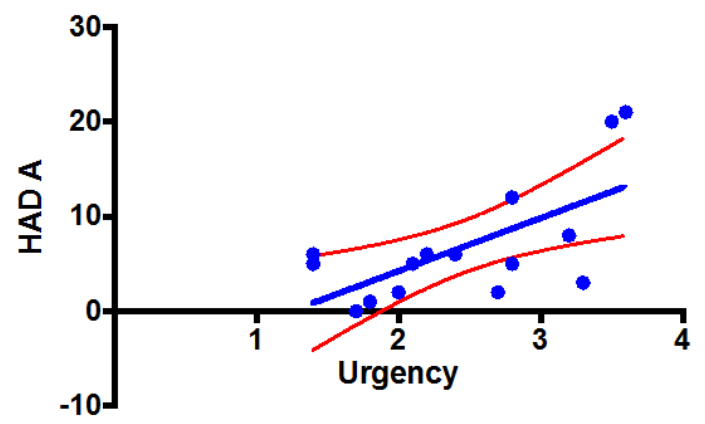

Figure 2. Shows the linear regression between urgency and HAD A, Y = 5,592*X - 6,957.

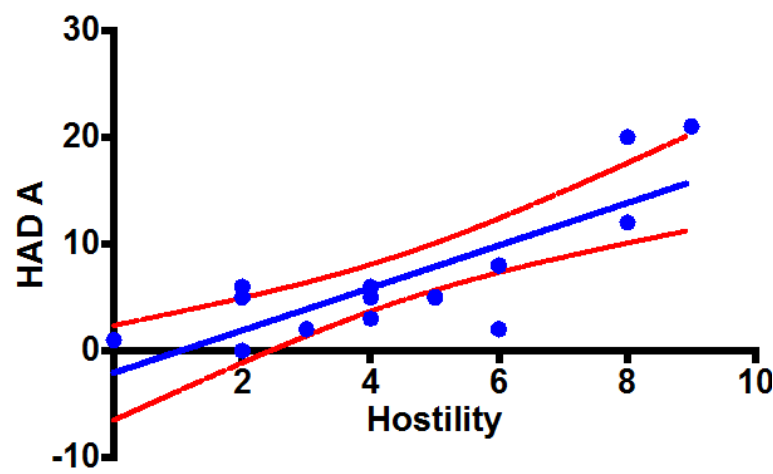

(a)

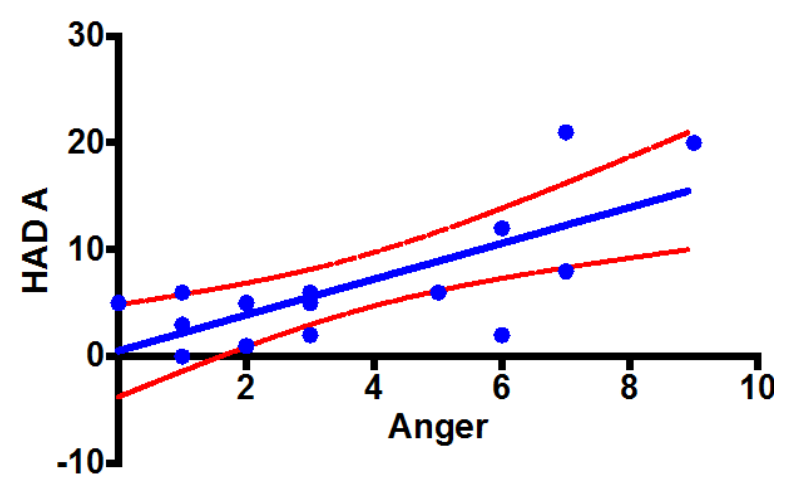

(b)

Figure 3. (a) Shows the linear regression between hostility and HAD A, Y = 1,988*X - 2,091 and (b) shows the linear regression between anger and HAD A, Y $=1,679 * X+0.5311$.

above the cut-off score of $\geq 8$ for depression. Only a small proportion of the inmates therefore showed levels of anxiety and depression that would normally elicit follow-up of the clinical state.

\section{Discussion}

Brutality and violent aggression represent a menace to society. Following blind and unpredicted violence, the questions of whether it could have been predicted and therefore avoided are frequently asked. In forensic psychiatry, the question of whether an aggressive act can be classified as impulsive or not is rarely met with an an- 
swer without reservations. The link between aggression and impulsivity is not clear, but there are studies describing an association between the two [10] [15]. The findings of the present study in extremely violent and brutal criminals do not confirm that higher impulsivity scores are associated with higher levels of reported physical aggression [15]. Our findings do, however, show that there is a significant relationship between impulsivity and aggression as demonstrated by the impulsivity subscale urgency and the aggression subscales hostility and anger in the two assessment questionnaires we used. Furthermore, the level of urgency among our violent criminals is significantly greater than in controls, indicating that individuals in our highly selected study group may carry a higher risk of committing violent acts because of the level of urgency. The difference between the two current study groups’ aggression scores for hostility and anger only just achieved statistical significance, possibly influenced by factors such as low sample size and lack of homogeneity. It must also be borne in mind that our population of inmates is different from the populations for whom the questionnaire is developed. Bryant and Smith based their study on 5 samples of undergraduates of both genders [37].

The UPPS is constructed based on information from 437 undergraduates, mostly women enrolled in an introductory psychology course [34] and has demonstrated its usefulness as a predictor as well as a potential tool for distinguishing between certain forms of aggression [39]. The present significant relationship between urgency and hostility supports observations showing a link between negative urgency and hostile attributes [40] and studies concluding that negative urgency may account for a significant variance in aggression [41]. No significant relationships between UPPS facets and aggressive responses are, however, found in a more recent study [42] examining the relationship between the UPPS facets of impulsivity and aggressive responses from a response choice aggression paradigm in college undergraduates.

We also observed a statistically significant relationship between impulsivity, i.e. urgency and symptoms of anxiety, and linear regression analysis shows a direct link between anxiety and urgency, but not between anxiety and the other UPPS impulsivity measures. Some studies have shown that patients with anxiety disorders without a lifetime history of comorbid major mood episodes have greater trait and state impulsivity than controls [43]. In conditions with substance use disorders, others have shown that the total scores on the Barrat Impulsivity Scale (BIS) [11] [44] may predict violence [45]. Furthermore, anxiety symptoms seem to account for the link between reactive aggression and sleep problems among Latino adolescents [46]. Taken together, there is growing evidence for the existence of subgroups of aggression, which in some anxiety and impulsivity levels may identify at-risk behavior [27]. Complicating matters further, subtypes of social anxiety disorder (SAD) have been recognized [47].

In our study, we also find that the inmates' scores on the aggression subscales hostility and anger, but not physical and verbal aggression, correlate with their scores for symptoms of anxiety on the HAD A subscale. No statistical significance is found when testing the relationship between the inmates' scores on the aggression subscales against HAD D symptoms of depression. A link between anxiety and aggression as indicated by the present results represents a platform shared with some previous observations, e.g. that persons with an angry or anxious mood state display a more aggressive attitude than those with a neutral mood [20], and with the observation of a correlation between the duration of anxiety and anger and hostility in patients with panic disorder [48].

We are unable to offer an explanation for the lack of correlation between the inmates' symptoms scores for anxiety and scores for physical or verbal aggression in this study, but a lack of clinically significant anxiety cannot be ruled out. A relationship between anxiety and aggression may not necessarily be direct as indicated by studies showing that anxiety symptoms seem to account for the link between reactive aggression and sleep problems [46] and that anxiety may act as a mediator in some cases of physical aggression [14].

Although our results are vulnerable to interfering factors, the chosen approach has resulted in what we believe is a strength of this study. To a certain extent the results reflect and support the existence of aggressive subgroups as described elsewhere. In this case an extremely brutal and aggressive group with the personality characteristics urgency, hostility and anger.

A complicating factor when identifying possible subgroups is that different studies often refer to the use of different scales applied on different populations, which impedes direct comparison. Different scales imply that different issues may have been addressed in the construction of the scales with direct consequences for what is actually measured. Applied to our highly selected population, the respective scales most likely contain different interfering biases. Therefore, beyond pointing in a direction which we believe is worth pursuing in future research, we are at present not comfortable with making more firm conclusions based on the results of our linear regression analyses. 
In studies like ours, there will always be questions regarding the validity of the results, especially when based on an individual's self-scores. The fact that all inmates included in the study population classed their childhood as good is of concern, since we have gathered strong evidence over the years that childhood trauma represents a risk factor for the development of aberrant behaviour later in life. Childhood physical punishment (CPP) has been associated with a number of negative psychosocial outcomes in adulthood [49], some of which are increased aggressiveness [50] [51], phobias, anxiety, schizotypal personality, and alcohol and drug abuse [52]. In view of the inmates' positive assessments on their childhoods, future research should also consider the possible interference of sublimation, a defence mechanism where socially unacceptable impulses are consciously transformed into socially acceptable actions. It is known that acting out and less use of sublimation predicts higher levels of anxiety [53] and that CPP may cause internalized anger and negativity towards others [49].

Some of the limitations of this study have already been addressed. The majority of the inmates included reported use of both legal and illegal drugs and stimulants. We were unable to gather detailed information on this to create a group-based overview. Classification of the aggressive acts based on information from the inmates makes it possible to deduce that only one of the severe criminal types of behaviour described could be classified as premeditated aggression, and the rest as impulsive. Strengths of our study were that the group of inmates included was relatively homogenous from a crime severity point of view-all having shown their potential for brutality.

To conclude, we see growing evidence suggesting that the use of subscales as in this study would be of benefit when trying to distinguish between different forms of aggression and impulsivity.

\section{Acknowledgements}

HV thanks Alvhilde Eliassens Research Foundation for financial support. The authors thank Alistair Reeves for help with the language.

\section{References}

[1] Gorodetsky, E., et al. (2014) The Interactive Effect of MAOA-LPR Genotype and Childhood Physical Neglect on Aggressive Behaviors in Italian Male Prisoners. Genes, Brain and Behavior, 13, 543-549. http://dx.doi.org/10.1111/gbb.12140

[2] Pardini, D.A., et al. (2014) Lower Amygdala Volume in Men Is Associated with Childhood Aggression, Early Psychopathic Traits, and Future Violence. Biological Psychiatry, 75, 73-80. http://dx.doi.org/10.1016/j.biopsych.2013.04.003

[3] Platje, E., et al. (2013) Longitudinal Associations in Adolescence between Cortisol and Persistent Aggressive or Rule-Breaking Behavior. Biological Psychology, 93,132-137. http://dx.doi.org/10.1016/j.biopsycho.2013.01.002

[4] Norman, R.E., et al. (2014) Trait Anxiety Moderates the Relationship between Testosterone Responses to Competition and Aggressive Behavior. Adaptive Human Behavior and Physiology, 1-13.

[5] American Psychiatric Association, W.D. (1952) Diagnostic and Statistical Manual of Mental Disorders.

[6] Coccaro, E.F. (2012) Intermittent Explosive Disorder as a Disorder of Impulsive Aggression for DSM-5. American Journal of Psychiatry, 169, 577-588. http://dx.doi.org/10.1176/appi.ajp.2012.11081259

[7] Dorfman, H.M., Meyer-Lindenberg, A. and Buckholtz, J.W. (2014) Neurobiological Mechanisms for Impulsive-Aggression: The Role of MAOA.

[8] Gina, G., et al. (2014) Proactive and Reactive Aggression and Cognitive and Affective Empathy among Students in Middle Childhood. International Journal of School and Cognitive Psychology, 1, 2.

[9] Swogger, M.T. et al. (2014) Impulsive versus Premeditated Aggression in the Prediction of Violent Criminal Recidivism. Aggressive Behavior.

[10] Stanford, M.S., Greve, K.W. and Dickens Jr., T.J. (1995) Irritability and Impulsiveness: Relationship to Self-Reported Impulsive Aggression. Personality and Individual Differences, 19, 757-760. http://dx.doi.org/10.1016/0191-8869(95)00144-U

[11] Barratt, E.S., Stanford, M.S., Dowdy, L., Liebman, M.J. and Kent, T.A. (1999) Impulsive and Premeditated Aggression: A Factor Analysis of Self-Reported Acts. Psychiatry Research, 86, 163-173. http://dx.doi.org/10.1016/S0165-1781(99)00024-4

[12] Stanford, M.S., Houston, R.J., Mathias, C.W., Villemarette-Pittman, N.R., Helfritz, L.E. and Conklin, S.M. (2003) Characterizing Aggressive Behavior. Assessment, 10, 183-190. http://dx.doi.org/10.1177/1073191103010002009 
[13] Perugi, G., Del Carlo, A., Benvenuti, M., Fornaro, M., Toni, C., Akiskal, K., Dell’Osso, L. and Akiskal, H. (2011) Impulsivity in Anxiety Disorder Patients: Is It Related to Comorbid Cyclothymia? Journal of Affective Disorders, 133, 600-606. http://dx.doi.org/10.1016/j.jad.2011.04.033

[14] Arnocky, S., Sunderani, S., Gomes, W. and Vaillancourt, T. (2014) Anticipated Partner Infidelity and Men’s Intimate Partner Violence: The Mediating Role of Anxiety. Evolutionary Behavioral Sciences, 9, 186-196.

[15] Hatfield, J. and Dula, C.S. (2014) Impulsivity and Physical Aggression: Examining the Moderating Role of Anxiety. The American Journal of Psychology, 127, 233-243. http://dx.doi.org/10.5406/amerjpsyc.127.2.0233

[16] Kashdan, T.B., Collins, R.L. and Elhai, J.D. (2006) Social Anxiety and Positive Outcome Expectancies on Risk-Taking Behaviors. Cognitive Therapy and Research, 30, 749-761. http://dx.doi.org/10.1007/s10608-006-9017-x

[17] Kashdan, T.B., McKnight, P.E., Richey, J.A. and Hofmann, S.G. (2009) When Social Anxiety Disorder Co-Exists with Risk-Prone, Approach Behavior: Investigating a Neglected, Meaningful Subset of People in the National Comorbidity Survey-Replication. Behaviour Research and Therapy, 47, 559-568. http://dx.doi.org/10.1016/j.brat.2009.03.010

[18] Kashdan, T.B. and McKnight, P.E. (2010) The Darker Side of Social Anxiety: When Aggressive Impulsivity Prevails over Shy Inhibition. Current Directions in Psychological Science, 19, 47-50. http://dx.doi.org/10.1177/0963721409359280

[19] Howell, A.N., Buckner, J.D. and Weeks, J.W. (2014) Culture of Honour Theory and Social Anxiety: Cross-Regional and Sex Differences in Relationships among Honour-Concerns, Social Anxiety and Reactive Aggression. Cognition and Emotion, 29, 568-577.

[20] Nederlof, A.F., Muris, P. and Hovens, J.E. (2014) Anger, Anxiety, and Feelings of Delusional Threat as Predictors of Aggressive Attitudes: An Experimental Mood Induction Study in a Non-Clinical Sample. Personality and Individual Differences, 57, 25-30. http://dx.doi.org/10.1016/j.paid.2013.09.006

[21] Gray, J.A. (1987) The Psychology of Fear and Stress. CUP Archive, Cambridge.

[22] Dickman, S.J. (1990) Functional and Dysfunctional Impulsivity: Personality and Cognitive Correlates. Journal of Personality and Social Psychology, 58, 95-102. http://dx.doi.org/10.1037/0022-3514.58.1.95

[23] Wallace, J.F., Newman, J.P. and Bachorowski, J.-A. (1991) Failures of Response Modulation: Impulsive Behavior in Anxious and Impulsive Individuals. Journal of Research in Personality, 25, 23-44. http://dx.doi.org/10.1016/0092-6566(91)90003-9

[24] Zuckerman, M., Kuhlman, D.M. and Thornquist, M. (1991) Five (or Three) Robust Questionnaire Scale Factors of Personality without Culture. Personality and Individual Differences, 12, 929-941. http://dx.doi.org/10.1016/0191-8869(91)90182-B

[25] Newman, J.P. and Wallace, J.F. (1993) Diverse Pathways to Deficient Self-Regulation: Implications for Disinhibitory Psychopathology in Children. Clinical Psychology Review, 13, 699-720. http://dx.doi.org/10.1016/S0272-7358(05)80002-9

[26] Kraepelin, E. and Defendorf, A.R. (1904) Clinical Psychiatry: A Text-Book for Students and Physicians: Abstracted and Adapted from the 6th German Edition of Kraepelin’s “Lehrbuch Der Psychiatrie”. Macmillan, London.

[27] Askénazy, F.L., Sorci, K., Benoit, M., Lestideau, K., Myquel, M. and Lecrubier, Y. (2003) Anxiety and Impulsivity Levels Identify Relevant Subtypes in Adolescents with At-Risk Behavior. Journal of Affective Disorders, 74, $219-227$. http://dx.doi.org/10.1016/S0165-0327(02)00455-X

[28] Barratt, E.S. (1993) Impulsivity: Integrating Cognitive, Behavioral, Biological, and Environmental Data.

[29] Gerbing, D.W., Ahadi, S.A. and Patton, J.H. (1987) Toward a Conceptualization of Impulsivity: Components across the Behavioral and Self-Report Domains. Multivariate Behavioral Research, 22, 357-379. http://dx.doi.org/10.1207/s15327906mbr2203_6

[30] Patton, J.H., Stanford, M.S. and Barratt, E.S. (1995) Factor Structure of the Barratt Impulsiveness Scale. Journal of Clinical Psychology, 51, 768-774. http://dx.doi.org/10.1002/1097-4679(199511)51:6<768::AID-JCLP2270510607>3.0.CO;2-1

[31] Stanford, M.S. and Barratt, E.S. (1992) Impulsivity and the Multi-Impulsive Personality Disorder. Personality and Individual Differences, 13, 831-834. http://dx.doi.org/10.1016/0191-8869(92)90057-V

[32] Zuckerman, M. (1994) Behavioral Expressions and Biosocial Bases of Sensation Seeking. Cambridge University Press, Cambridge.

[33] Costa Jr., P.T. and McCrae, R.R. (1990) Personality Disorders and the Five-Factor Model of Personality. Journal of Personality Disorders, 4, 362-371. http://dx.doi.org/10.1521/pedi.1990.4.4.362

[34] Whiteside, S.P. and Lynam, D.R. (2001) The Five Factor Model and Impulsivity: Using a Structural Model of Personality to Understand Impulsivity. Personality and Individual Differences, 30, 669-689. http://dx.doi.org/10.1016/S0191-8869(00)00064-7 
[35] Buss, A.H. and Perry, M. (1992) The Aggression Questionnaire. Journal of Personality and Social Psychology, 63, 452-459. http://dx.doi.org/10.1037/0022-3514.63.3.452

[36] Prochazka, H. and Ågren, H. (2001) Aggression in the General Swedish Population, Measured with a New Self-Rating Inventory: The Aggression Questionnaire-Revised Swedish Version (AQ-RSV). Nordic Journal of Psychiatry, 55, 17-23. http://dx.doi.org/10.1080/080394801750093661

[37] Bryant, F.B. and Smith, B.D. (2001) Refining the Architecture of Aggression: A Measurement Model for the BussPerry Aggression Questionnaire. Journal of Research in Personality, 35, 138-167. http://dx.doi.org/10.1006/jrpe.2000.2302

[38] Zigmond, A.S. and Snaith, R.P. (1983) The Hospital Anxiety and Depression Scale. Acta Psychiatrica Scandinavica, 67, 361-370. http://dx.doi.org/10.1111/j.1600-0447.1983.tb09716.x

[39] Derefinko, K., DeWall, C.N., Metze, A.V., Walsh, E.C. and Lynam, D.R. (2011) Do Different Facets of Impulsivity Predict Different Types of Aggression? Aggressive Behavior, 37, 223-233. http://dx.doi.org/10.1002/ab.20387

[40] Lynam, D.R. and Miller, J.D. (2004) Personality Pathways to Impulsive Behavior and Their Relations to Deviance: Results from Three Samples. Journal of Quantitative Criminology, 20, 319-341. http://dx.doi.org/10.1007/s10940-004-5867-0

[41] Miller, J., Flory, K., Lynam, D. and Leukefeld, C. (2003) A Test of the Four-Factor Model of Impulsivity-Related Traits. Personality and Individual Differences, 34, 1403-1418. http://dx.doi.org/10.1016/S0191-8869(02)00122-8

[42] Seibert, L.A., Miller, J.D., Pryor, L.R., Reidy, D.E. and Zeichner, A. (2010) Personality and Laboratory-Based Aggression: Comparing the Predictive Power of the Five-Factor Model, BIS/BAS, and Impulsivity across Context. Journal of Research in Personality, 44, 13-21. http://dx.doi.org/10.1016/j.jrp.2009.09.003

[43] Del Carlo, A., Benvenuti, M., Fornaro, M., Toni, C., Rizzato, S., Swann, A.C., Dell’Osso, L. and Perugi, G. (2012) Different Measures of Impulsivity in Patients with Anxiety Disorders: A Case Control Study. Psychiatry Research, 197, 231-236. http://dx.doi.org/10.1016/j.psychres.2011.09.020

[44] Barratt, E.S. (1959) Anxiety and Impulsiveness Related to Psychomotor Efficiency. Perceptual and Motor Skills, 9, 191-198. http://dx.doi.org/10.2466/pms.1959.9.3.191

[45] Zhou, J., Witt, K., Chen, C., Zhang, S., Zhang, Y., Qiu, C., Cao, L. and Wang, X. (2014) High Impulsivity as a Risk Factor for the Development of Internalizing Disorders in Detained Juvenile Offenders. Comprehensive Psychiatry, 55, 1157-1164. http://dx.doi.org/10.1016/j.comppsych.2014.03.022

[46] Fite, P.J., Becker, S.P., Rubens, S.L. and Cheatham-Johnson, R. (2014) Anxiety Symptoms Account for the Link between Reactive Aggression and Sleep Problems among Latino Adolescents. Child \& Youth Care Forum, 44, 343-354.

[47] Mortberg, E., Tillfors, M., van Zalk, N. and Kerr, M. (2014) An Atypical Anxious-Impulsive Pattern of Social Anxiety Disorder in an Adult Clinical Population. Scandinavian Journal of Psychology, 55, 350-356. http://dx.doi.org/10.1111/sjop.12117

[48] Sugaya, N., Yoshida, E., Yasuda, S., Tochigi, M., Takei, K., Otowa, T., et al. (2015) Anger Tendency May Be Associated with Duration of Illness in Panic Disorder. BioPsychoSocial Medicine, 9, 6. http://dx.doi.org/10.1186/s13030-015-0035-3

[49] Björkqvist, K. and Österman, K. (2014) Does Childhood Physical Punishment Predispose to a "Victim Personality”. Pediatrics \& Therapeutics, 4, 190.

[50] Gershoff, E.T. (2002) Corporal Punishment by Parents and Associated Child Behaviors and Experiences: A MetaAnalytic and Theoretical Review. Psychological Bulletin, 128, 539-579. http://dx.doi.org/10.1037/0033-2909.128.4.539

[51] Straus, M.A. (1991) Discipline and Deviance: Physical Punishment of Children and Violence and Other Crime in Adulthood. Social Problems, 38, 133-154. http://dx.doi.org/10.2307/800524

[52] Afifi, T.O., Mota, N.P., Dasiewicz, P., MacMillan, H.L. and Sareen, J. (2012) Physical Punishment and Mental Disorders: Results from a Nationally Representative US Sample. Pediatrics, 130, 184-192. http://dx.doi.org/10.1542/peds.2011-2947

[53] Jun, J.Y., Lee, Y.-J.G., Lee, S.-H., Yoo, S.Y., Song, J. and Kim, S.J. (2015) Association between Defense Mechanisms and Psychiatric Symptoms in North Korean Refugees. Comprehensive Psychiatry, 56, 179-187.

http://dx.doi.org/10.1016/j.comppsych.2014.10.001 